ARTÍCULO DE INVESTIGACIÓN

\title{
CURSO CLÍNICO Y PERFIL NEUROPSICOLÓGICO DE UN CASO DE TRASTORNO NEUROCOGNITIVO FRONTOTEMPORAL MAYOR VARIANTE LINGÜÍSTICA
}

\author{
CLINICAL COURSE AND NEUROPSYCHOLOGICAL PROFILE OF FRONTOTEMPORAL \\ NEUROCOGNITIVE DISORDER LINGUISTIC LANGUAGE VARIANT.
}

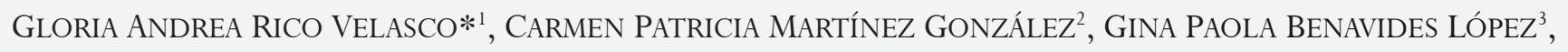 \\ MARIBEL MOTTA ARTUNDUAGA \\ UNIVERSIDAD CATÓLICA LUIS AMIGÓ \\ INSTITUTO LATINOAMERICANO DEL SISTEMA NERVIOSO- ILANS \\ MOBILITY GROUP \\ FECHA RECEPCIÓN: 22/02/2021 • FECHA ACEPTACIÓN: 15/06/2021 \\ Para citar este artículo: Rico, G., Martínez, C., Benavides, L., \& Motta, M. (2021). Curso clínico y perfil neuropsicológico de un caso \\ de trastorno neurocognitivo frontotemporal mayor variante lingüística. \\ Psychologia, 15(1), 57-67. https://doi: 10.21500/19002386.5281
}

\begin{abstract}
Resumen
El trastorno neurocognitivo frontotemporal es una enfermedad neurodegenerativa que incluye manifestaciones clínicas de subtipo comportamental y lingüística. La afasia progresiva primaria (APP) es un síndrome en el que aparecen alteraciones del lenguaje que comprende tres tipos de variantes: no fluente, semántica y logopénica. Este estudio describe la evolución clínica y las características neuropsicológicas de una mujer de 63 años que presenta un deterioro progresivo del lenguaje. Se evalúan las funciones de atención, memoria, lenguaje y funciones ejecutivas. La paciente obtuvo un bajo rendimiento en memoria, velocidad de procesamiento y funciones ejecutivas. Su lenguaje se caracteriza por presentar baja fluidez, agramatismo, parafasias verbales y dificultades en denominación. Se concluye que la paciente presenta características de la APP no fluente, que varía a través del tiempo y afecta su funcionamiento; características de un curso clínico de un trastorno neurocognitivo mayor posible debido a una degeneración del lóbulo frontotemporal.
\end{abstract}

Palabras Clave: afasia progresiva primaria, neuropsicología, cognición, deterioro cognitivo, Demencia Frontotemporal.

1* Universidad Católica Luis Amigó. ORCID: https://orcid.org/0000-0002-6980-19011. gloria.ricove@amigo.edu.co

2 Instituto Latinoamericano del Sistema Nervioso (Ilans). ORCID: https://orcid.org/0000-0001-5375-1666

3 Mobility Group. ORCID: https://orcid.org/0000-0002-5054-6533 


\begin{abstract}
Mild cognitive impairment, frontotemporal dementia (FTD) is a neurodegenerative disease characterized by clinical manifestations of behavior and linguistic subtypes. Primary Progressive Aphasia (APP) is a syndrome in which language alterations appear that include three types of variations: Non - fluent, Semantic and Logopenic. This study describes the clinical evolution and the neurophysiological characteristics of a 63 years old woman that started with a progressive language impairment. The functions which are evaluated are attention, memory, language and executive functions. The patient obtained a low performance in memory, processing speed and executive functions. The language is characterized by low fluency, agramatism, paraphasias and denomination difficulties. It is concluded, that the patient has characteristics of APP non-fluent which varies throughout the time and it affects her performance; characteristics of a clinical course of a greater neurocognitive disorder might be due to a lobe frontotemporal degeneration. Key words: Primary progressive aphasia, neuropsychology, cognition, cognitive impairment, frontotemporal dementia.

\section{Introducción}

Los trastornos neurocognitivos se caracterizan por un declive en las funciones mentales superiores como la memoria, la atención, el lenguaje y funciones ejecutivas, comparado con el nivel previo de funcionamiento que conlleva a una afectación en la dependencia funcional del individuo (Rohan \& Matej, 2014). Estos déficits son de diversas etiologías, como la enfermedad de Alzheimer, la enfermedad cerebrovascular, la enfermedad de Parkinson, o la demencia frontotemporal (DFT), entre otros.

La DFT describe un espectro de desórdenes clíni-

conocida, que se presenta en individuos entre los $45 \mathrm{y}$ 65 años de edad (Seltman \& Mattews, 2012), tiene una representación aproximada del $20 \%$ dentro de las demencias (Onyike \& Diehl-Schmid, 2013). Según el Manual Diagnóstico de los Desórdenes Mentales, DSM 5 (APA, 2014), Tabla 1, se define el trastorno neurocognitivo (TNC) frontotemporal como un síndrome que incluye una variable del comportamiento y otra del lenguaje (semántica, agramatical/no fluente y logopénica), asociado a la atrofia e hipometabolismo de los lóbulos frontales y temporales, con relativa preservación de las regiones cerebrales posteriores (Rohan \& Matej, 2014).
\end{abstract} cos, patológicos y neurodegenerativos, de etiología des-

Tabla 1. Criterios Diagnósticos para el trastorno neurocognitivo mayor o leve debido a DLFT. DSM 5 (APA, 2014)

Trastorno neurocognitivo frontotemporal mayor o leve

A. Se cumplen los criterios de un trastorno neurocognitivo mayor o leve.

B. El trastorno presenta inicio insidioso y progresión gradual.

C. Aparece (1) o (2)

1. Variante de comportamiento

a. Tres o más síntomas:

I. Desinhibición.

II. Apatía e inercia.

III. Pérdida de simpatía y empatía.

IV. Comportamiento conservado, estereotipado, compulsivo o ritualista.

V. Hiperoralidad y cambios dietéticos

b. Declive destacado de la cognición social o de las capacidades ejecutivas

2. Variante del lenguaje.

a. Declive destacado de la habilidad para usar el lenguaje, ya sea en producción del habla, elección de las palabras, denominación de objetos, gramática o comprensión de las palabras

D. Ausencia relativa de las funciones perceptuales, motora, de aprendizaje y memoria

E. La alteración no se explica por una enfermedad cerebrovascular, otra enfermedad neurodegenerativa, los efectos de una sustancia o algún otro trastorno mental, neurológico o sistémico. 
Los cambios presentados en la variante comportamental se asocian con una atrofia de la corteza frontal medial anterior, ínsula cíngulo anterior y lóbulo temporal anterior (Acosta, Canu, Sarro, Comi \& Filipi, 2012). Dentro de la variante del lenguaje se encuentra la afasia progresiva primaria (APP), que se define como una alteración en el lenguaje de aparición progresiva, causada por enfermedad neurodegenerativa, durante al menos los 2 primeros años, estando el resto de los dominios cognitivos preservados, y que interfiere con las actividades de la vida diaria por la disfunción del lenguaje (Gorno-Tempini et al., 2011).

Dentro de la APP se describen tres variantes: la APP no fluente o agramática, la APP variante semántica y la APP logopénica, cuyas características y criterios diagnósticos fueron descritas por Gorno-Tempini et al. (2011). En la fase preclínica, los pacientes con APP pueden manifestar más esfuerzos para expresarse; a medida que la condición progresa, presentan bajo rendimiento en tareas de denominación, fluidez verbal, sintaxis o descripción de imágenes, manteniendo una buena ejecución en otros dominios cognitivos y un buen funcionamiento en actividades básicas y complejas de la vida diaria, aspecto que se deteriora de forma más tardía que en otras demencias, progresa entre 7 y 10 años, evolucionando hasta la "demencia afásica" y el mutismo (Matías-Guiu \& García-Ramos, 2013; Dickerson, 2011; Sapolsky et al., 2014).

\section{Variante no fluente o agramatical (APPvnf)}

Se caracteriza por un lenguaje espontáneo lento y laborioso, telegráfico, con la característica principal de agramatismo, parafasias fonémicas, en ocasiones evidente dificultad en la secuenciación de los movimientos necesarios para una correcta producción del habla, considerada como apraxia del habla, progresando a fallos en la construcción y sintaxis de frases, mayor esfuerzo para la utilización de verbos, además, dificultad en repetición de frases largas y palabras multisilábicas, acalculia, apraxia ideomotora y orofacial, sin presentarse déficits sensoriales ni motores y que no es generada por accidente cerebrovascular, infecciones, lesiones, hidrocefalia y otros, muy similar a la afasia de Broca (Grossman, 2012; Harciarek \& Kertesz, 2011).

Por otra parte, conservan de la inteligencia no verbal y la memoria (Kertész \& Muñoz, 1998); se conside- ra que presenta un curso estable y de progresión lenta. Está asociada con atrofia en región posterior fronto-insular, observada a través de resonancia magnética (MRI), SPECT y PET y en relaciones histopatológicas; se ha encontrado en la DFT la presencia de proteína tau. (Harris \& Jones, 2014; Grossman, 2012).

\section{Variante semántica (APPvS)}

Anteriormente denominada demencia semántica (Snowden, 1998 citado por Harciarek \& Kertesz, 2011), se caracteriza por la pérdida progresiva y multimodal del conocimiento semántico, anomia, pérdida del significado de las palabras, con un vacío de contenido, presencia de circunloquios y parafasias; por otra parte, el lenguaje es fluente, espontáneo, con adecuada repetición, prosodia, fonológica y gramaticalmente correcto. La comprensión puede estar conservada, pero con incapacidad para definir palabras simples; se percibe dificultad para seguir instrucciones que involucran palabras de baja frecuencia y que progresa a una dificultad comprensiva debido a la pérdida de significado de palabras comunes (Grossman, 2012).

La evolución clínica se presenta con una progresión de una afasia anómica (fase inicial) a una afasia transcortical sensorial (fase intermedia) y, finalmente, como una afasia global grave (fase final) (Torres \& López, 2011). A nivel neuroanatómico, SPECT y PET muestran atrofia, hipometabolismo o hipoperfusión en área anterior del lóbulo temporal anterior. La histopatología de DLFT con presencia de TDP-43 (Harris \& Jones, 2014; Arnedo, Bembibre \& Triviño, 2013) se ha asociado con cambios en la vía ventral, específicamente en el fascículo uncinado y el fascículo longitudinal inferior, el área del fascículo arqueado del fascículo longitudinal superior.

\section{Variante Logopénica de la APP (APPvl)}

La variante logopénica se caracteriza por un lenguaje lento, no fluente, con frecuentes pausas para encontrar las palabras, con presencia de parafasias fonológicas, con alteración en la capacidad de repetición de frases largas; pero, a diferencia de la APPvnf, conserva la habilidad motora del lenguaje oral, la gramática y la comprensión de las palabras, relaciones semánticas, no presentan agramatismos ni lenguaje telegráfico (Grossman, 2010; Gorno-Tempini et al., 2011).

La evolución clínica de este tipo de trastorno tiene características de una afasia de conducción, el declive en 
el lenguaje y funciones cognitivas es rápido, con un promedio de deterioro de 18 meses (Magnin et al., 2015). Los pacientes con APPvl presentan fallos en la memoria episódica anterógrada cuando subyace la EA.

Se ha observado que en estudios con uso de SPECT y PET muestran hipometabolismo o hipoperfusión de región parietal, perisilviana posterior izquierda, así como histopatología heterogénea relacionado con EA (Harris \& Jones, 2014; Arnedo, Bembibre \& Triviño, 2013).

En la evaluación para el diagnóstico neuropsicológico de la APP se deben tener en cuenta aspectos como la progresión del cuadro clínico de la APP, el perfil cognitivo, el grado de funcionalidad del paciente que afecte las habilidades básicas y una evaluación específica del lenguaje para establecer un diagnóstico diferencial con respecto a otros procesos neurodegenerativos (Arnedo, Bembibre \& Triviño, 2013).

Este estudio tiene como objetivo describir la evolución del perfil neuropsicológico de una paciente de 63 años con posible DFT de variante lingüística, conocida por el servicio de neuropsicología de la Dirección de Sanidad de la Policía Nacional desde el 2012 hasta el 2017.

\section{Método}

\section{Diseño}

Estudio descriptivo de caso único que especifica sus propiedades y características (Hernández, Fernández \& Baptista, 2010).

\section{Descripción del caso}

Paciente (IA), sexo femenino, edad 63 años, procedente de Bogotá, Bacterióloga cesante, casada, lateralidad diestra, con antecedente médico de síndrome de Sjögren, hipotiroidismo e hiperlipidemia. Remitida al servicio de neuropsicología por neurología en el 2012. El reporte del neurólogo tratante describe que IA inicia con "problemas relacionados con el olvido del nombre de las cosas, tartamudez y bloqueos".

Según reporte actual de su esposo, IA inició con quejas de tartamudez en 2012, olvido del nombre de los objetos, se quedaba diciendo "quiero... mm... quiero... quiero”, pero sin lograr denominar la palabra que quería decir, junto con dificultad para recordar el nombre de alimentos, personas y lugares; "no sabe cómo se llama una gallina, no articula una frase”, describe su esposo.
Reconoce a las personas y los lugares de su pasado, pero es incapaz de nombrarlos. Actualmente, repite "si... si... si..." cuando intenta comenzar a hablar, saluda, se despide, da las gracias, expresa disgusto y emociones de forma no verbal (se va del lugar). Según su esposo "entiende perfectamente" lo que le dicen. En su personalidad previa la describe como "dinámica e inquieta"; ahora es temerosa, callada y pide repetir la información varias veces. No ha tenido cambios en su comportamiento, no reporta comportamientos ritualistas o estereotipados. Es independiente en las actividades de la vida diaria, en actividades básicas e instrumentales. No ha tenido eventos de desorientación espacial. Respecto a su memoria, se encuentra totalmente preservada. Por recomendación médica sale acompañada de su esposo. Su apariencia personal es impecable. Asiste a terapia por fisioterapia, del lenguaje y estimulación cognitiva desde hace 3 años.

El reporte de la primera remisión en 2012 concluye que: "La paciente presenta en la valoración neuropsicológica un perfil normal de acuerdo con su edad, con fallas de tipo atencional que, de acuerdo con lo reportado por el esposo, siempre las ha tenido".

Durante el 2015, presentó progresión en las funciones ejecutivas y primordialmente alteraciones del lenguaje expresivo y comprensivo a nivel verbal.

Fue evaluada por clínica de memoria en septiembre de 2015, donde se concluye que estos cambios en su función cognitiva se relacionan con una posible afasia progresiva primaria en etapas iniciales.

Se encuentra una RM cerebral simple, tomada el 14 de junio de 2015 (ver Figura 1), que registra ligera prominencia de surcos corticales, cisternas basales y sistema ventricular indicativo de atrofia cerebral.

En 2017 se realiza una nueva resonancia, evidenciando "un aumento en la amplitud del sistema ventricular, de los surcos del espacio subaracnoideo periférico y de las cisternas de la base, concluyendo que IA presenta atrofia cerebral difusa con predominio parietal”, figuras 1 y 2 .

Figura 1. A) IMR de IA corte sagital T1 mayo de 2017. B). C) Corte axial de IA en T1 lóbulos temporales, 2017. 

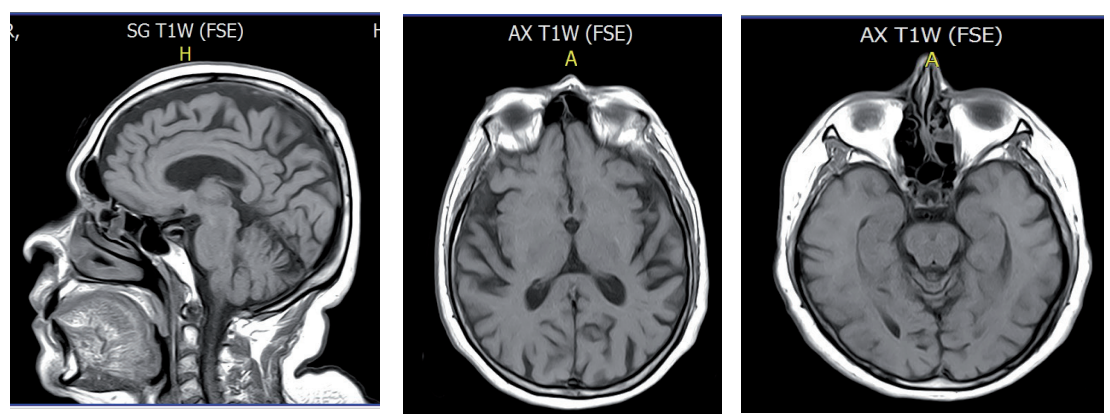

Figura 2. Corte coronal posterior IA 2015 y 2017

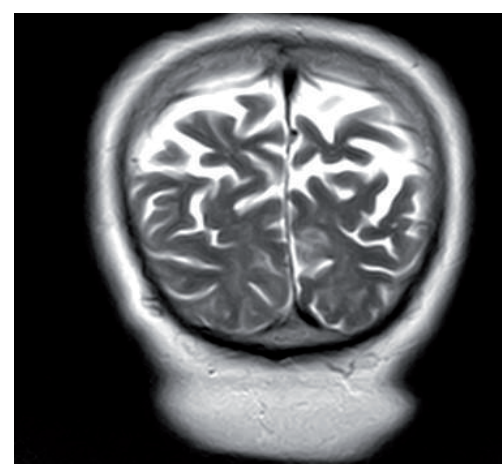

T1- 2015

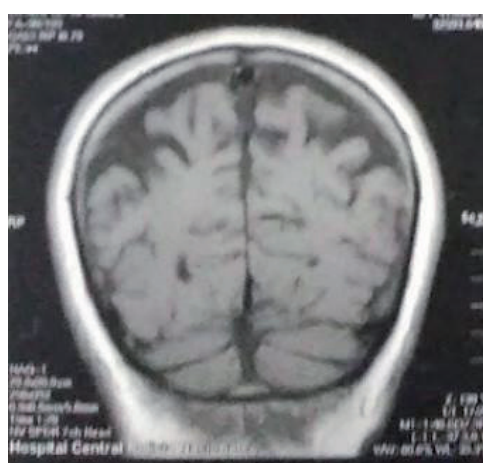

T2-2017

Instrumentos

Se realizó un protocolo de evaluación de las funciones cognitivas, usando pruebas específicas de lenguaje. Cada subprueba empleada se registra a continuación:

Tabla 3. Protocolo de evaluación funciones cognitivas

\begin{tabular}{ll}
\hline \hline Dominio & Prueba \\
\hline \hline Rastreo global & $\begin{array}{l}\text { Mini Examen del Estado Mental (MMSE). Folstein, Folstein y McHugh. } \\
\text { Adaptación española (Lobo et al, 2002). }\end{array}$ \\
Capacidad atencional y & Claves (WAIS) \\
velocidad de procesamiento & $\begin{array}{l}\text { Búsqueda de símbolos (WAIS) } \\
\text { Atención sostenida auditiva (Dígitos directos WAIS) }\end{array}$ \\
Atención sostenida visual & $\begin{array}{l}\text { Prueba de Rastreo o De Trazo A (Trail Making Test) Autor: Aaron Smith (Reitan \& } \\
\text { Wolfson, 1985). }\end{array}$ \\
Memoria Explícita Verbal & $\begin{array}{l}\text { Test Grober \& Buschke (Free and Cued Selective Remined Test (FCSRT), Grober, } \\
\text { Buschke, Crystal, Bang \& Dresner, 1988) }\end{array}$ \\
Memoria visual & Test de copia y reproducción de la Figura de Rey (Test de Copie et Reproductione de \\
Habilidades visuoespaciales & Subtest de Test de Barcelona (Peña-Casanova 1990).
\end{tabular}




\begin{tabular}{ll}
\hline \hline Dominio & Prueba \\
\hline \hline & Fluidez semántica y Fonológica Borkowski (Benton \& Spreen, 1967). \\
& Test de Barcelona (Peña-Casanova 1990). Para este caso se usaron las subpruebas \\
& de lenguaje espontáneo, narración temática, repetición de sílabas, \\
& pseudopalabras, repetición de palabras pares mínimos, repetición palabras, \\
& repetición frases. \\
& Vocabulario (WAIS). \\
& Test de Boston para el Diagnóstico de las Afasias (TBDA). (Goodglass \& Kaplan, \\
& 1978). \\
& Escala de Severidad en la Afasia Primaria Progresiva. PASS, Progressive Aphasia Severity \\
& Scale. (Sapolsky, Domoto-Reilly, Brickhouse, McGinnis \& Dickerson, 2014). \\
& Prueba de Rastreo o De Trazo B (Trail Making Test) (Reitan \& Wolfson, 1985). \\
& Subprueba de Semejanzas, de la Batería WAIS III (Wechsler, 2003). \\
& Subprueba dígitos de la Batería WAIS III (Wechsler, 2003). \\
& Aritmética (WAIS) \\
Lenguaje & Indice de Barthel (IB) (Barrero, 2005) \\
& Escala de Lawton \& Brody (Philadelphia Geriatric Center Instrumental Activities of Daily \\
& Living, 1969). \\
& Inventario de Quejas de Subjetivas de Memoria (QSM) (Marotto, 2003). \\
& Escala de depresión Geriátrica de Yesavage. (Brink, 1982). \\
Funciones ejecutivas & Inventario de Ansiedad de Beck BAI (Beck \& Steer, 2011). \\
Inventario comportamental frontal de Kertesz y Muñoz, (Kertesz et al., 2000). \\
Valoración Clínica de Demencia, CDR (Clinical Dementia Rating, Charles Hughes). \\
\hline \hline
\end{tabular}

\section{Procedimiento}

El presente estudio de caso incluye cuatro fases descritas a continuación.

Fase 1: Acceso y selección del estudio de caso; recolección de la información de la historia clínica en el sistema de registro de la Dirección de Sanidad de la Policía Nacional en Bogotá, Colombia. Entrevista semiestructurada con la paciente y su esposo, en la cual se recolectan datos sociodemográficos, historia del problema, antecedentes médicos, exámenes y tratamientos actuales. Se realiza la firma del consentimiento informado por parte de la paciente y su esposo.

Fase 2: Aplicación de instrumentos del estudio. Se realizaron 3 sesiones de aproximadamente una hora de duración. Se Inicia proceso de evaluación neuropsicológica que incluye el protocolo utilizado por el Departamento de Neuropsicología de la Institución de los instrumentos descritos anteriormente.
Fase 3: Análisis de datos Calificación e interpretación de la base de datos para su respectivo análisis, se usaron los manuales de los Test de Boston y Barcelona y los baremos de la población colombiana de pruebas neuropsicológicas (Arango \& Rivera, 2015), para describir el perfil neuropsicológico y entrega de informe de IA.

Fase 4: Análisis y comparación de resultados con las teorías encontradas y redacción del artículo.

\section{Consideraciones éticas}

Se redactó un consentimiento informado; fue leído y firmado por los participantes (paciente y familia), previamente a la aplicación de las pruebas. De acuerdo con la resolución 8430 de 1993 del Ministerio de Salud, se considera que esta investigación es de riesgo inferior al mínimo para los participantes; se garantizaron los derechos al anonimato, la confidencialidad, la no maleficencia y el beneficio de participar en la investigación. A cada uno de los participantes (paciente y familia) se les informó acerca del estado cognitivo global de la paciente mediante un informe escrito y específicamente se presentaron los resultados de la aplicación de los instrumentos. 


\section{Resultados}

La descripción de la ejecución de IA en las pruebas neuropsicológicas y su comparación con la evaluación de 2015 se describe a continuación y se puede apreciar visualmente en la Figura 3.

Estado cognitivo global. El MMSE se encuentra en el punto de corte, indicando normalidad. Este resultado es similar al de 2015.

Orientación. Conserva la orientación en tiempo y espacio.

Atención. En cuanto a la atención sostenida de la modalidad visual, se encuentra una ejecución normal (TMT-A), similar a la obtenida en 2015; en contraste, muestra una ejecución alterada en la atención sostenida del canal auditivo, así como se evidencia una mayor latencia de respuesta, lo que sugiere una disminución de la velocidad de procesamiento visual. Respecto a la atención dividida visual, se sitúa en un promedio bajo en prueba Claves y búsqueda de símbolos del WAIS.

Memoria. La memoria explícita verbal medida a través del Test de Grober \& Buschke; después de lectura y codificación a lo largo de 3 ensayos, IA arrojó una curva de aprendizaje improductiva, plana, con un bajo volumen de información respecto a lo esperado en edad y educación $(4,4,3)$. Esta curva, no mejora con la clave semántica $(2,1,3)$ y no logra ubicarse en la normalidad. En el recuerdo diferido, 20 minutos, las evocaciones libres (2) y con clave (1) son menores a lo esperado. Se encuentran errores patológicos de intrusiones y perseveraciones. Estos resultados evidencian una alta dificultad en los procesos de consolidación y evocación de la información verbal auditiva. Su rendimiento es menor en comparación con la última evaluación obtenida en el recuerdo inmediato y diferido, así como aumento de fenómenos patológicos en la actual evaluación.

En la memoria visual, evaluada a través de la evocación de la figura de Rey, IA se ubica en un rango por debajo de lo esperado.

Praxia constructiva. Logra construir la figura esperada, tipo IV, que evidencia una yuxtaposición de detalles, con déficit en integrar los detalles de forma global y con fallas en la planificación. Por otro lado, se observa mayor tiempo de ejecución en procesamiento de in- formación visual, comparado con última evaluación en 2015.

\section{Habilidades visoperceptivas.}

A nivel visuoespacial, IA evidencia normalidad en su desempeño, en el subtest imágenes superpuestas logra reconocer 19 imágenes de las 20 presentadas.

\section{Gnosias.}

Medida a través del reconocimiento de caras del test de Bercelona, IA no presenta alteraciones.

Funciones ejecutivas. Expone alteración en la abstracción de la información, conocimiento léxico y categorización verbal (refranes y semejanzas), asimismo, expone un déficit en la capacidad para hacer búsqueda activo-ejecutiva a partir de claves fonológicas y semánticas. Adicionalmente, se observan fallas en la atención alternante, con dificultad para intercambiar estímulos y presentando un aumento significativo en el tiempo de ejecución. La planeación se observa alterada, debido a la dificultad en organizar e implementar estrategias para copiar secuencialmente los elementos de una figura compleja. Respecto a la memoria de trabajo, IA refleja dificultad para invertir los dígitos solicitados. Disminuye su rendimiento comparado con evaluación de 2015 en abstracción y mayor tiempo de ejecución en alternancia.

Funcionalidad. En cuanto a las actividades básicas de la vida diaria (ABVD), IA es independiente en la realización de actividades básicas (Barthel) e instrumentales de la vida diaria (Lawton \& Brody). La escala PASS muestra una alteración significativa de las habilidades instrumentales afectadas por el uso del lenguaje. El deterioro cognitivo encontrado la ubica en un rango de Demencia Leve (CDR).

Socioemocional. Se evidencia que IA no presenta cambios de comportamiento sugestivos de la DFT, como apatía, desinhibición o hiperoralidad, de acuerdo con el Inventario de Comportamiento de Kertesz. A nivel emocional hay presencia de ansiedad leve, según la escala de valoración de Hamilton; no presenta signos de depresión, según la escala Yesavage. 
Figura 3. Evolución del perfil neuropsicológico de IA de los años 2012, 2015 y 2017.

\section{Evolución perfil neuropsicológico IA}

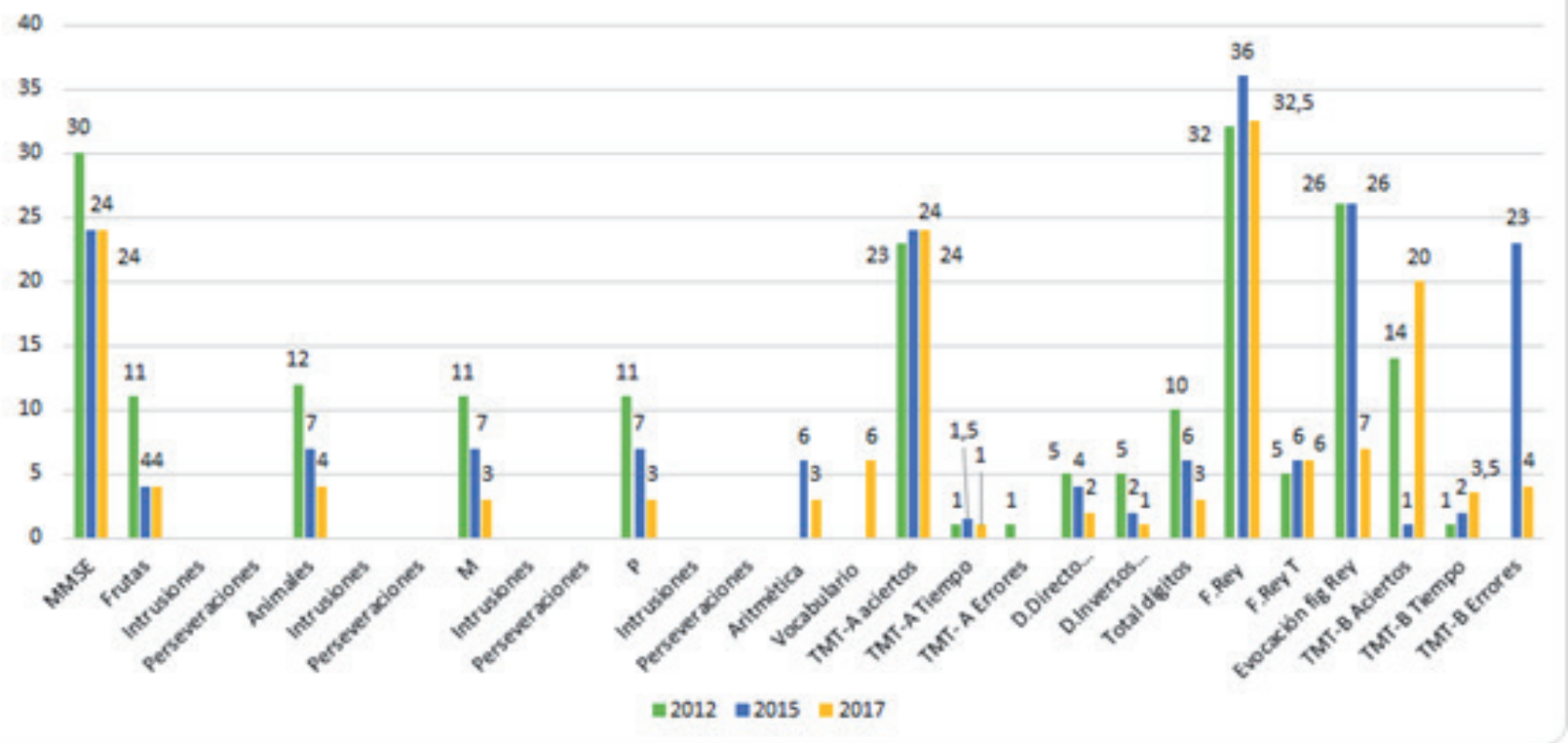

Nota: Los resultados muestran la puntuación directa para cada prueba excepto para las que componen la escala WAIS (aritmética, vocabulario y dígitos) que figuran en la puntuación escalar. La prueba descrita tomada en tiempo como el TMT- A y B se muestran en minutos.

\section{Evaluación Neuropsicológica Específica de Lenguaje.}

Las habilidades conservadas de IA son la comprensión auditiva, la repetición de material verbal simple, como letras, sílabas, pares de sílabas y pseudopalabras, la lectura, la escritura a la copia y al dictado. Igualmente, obtuvo una calificación promedio en longitud de la frase y secuencias automatizadas (días de la semana, meses del año, contar hasta 21), su denominación es efectiva por confrontación visual.

Los resultados en el TBDA exponen una reducción de la expresión oral, con evidente habla telegráfica, omisión de palabras de función, alteración de la sintaxis, bajo contenido informativo, fallas en línea melódica, la agilidad verbal, que conlleva a una alteración en la fluidez. En consideración a la articulación, se observa alteración de la repetición de palabras compuestas por más de 5 sílabas que empeora a medida que aumenta la complejidad, igualmente, en frases de baja probabilidad.

A nivel subléxico, IA presenta parafasias fonémicas de adiciones, errores mixtos, ecolalia y palabras de predilección. Respecto a la denominación, el TBDA presenta un resultado favorable en denominación por confronta- ción visual, aunque requiere más tiempo para responder. Se observa dificultad para acceder a la palabra, característica de anomia, llegando a usar circunloquios de tipo instrumental para hacer referencia a la palabra solicitada.

Se encuentra un déficit en el recitado, canto y ritmo, no logra completar refranes sencillos, la repetición de palabras y oraciones asociadas a un ritmo le generan significativa dificultad. A nivel semántico, se observa que IA conserva el reconocimiento de los significados asociados a diferentes categorías y conceptos, tiende a acertar en mayor medida a estímulos visuales en comparación a estímulos auditivos, asimismo, su desempeño es mejor cuando las categorías son supraordinadas que las subordinadas, aunque la diferencia no es significativa. Adicionalmente, se observan falsos positivos frecuentes, pero se analiza que son producto de fallos en monitoreo y producto de agotamiento y el esfuerzo que sí se percibe en la paciente.

\section{Discusión}

El presente estudio evalúa el caso de IA, mujer de 63 años, de escolaridad profesional, con antecedentes 
de hiperlipidemia, hipotiroidismo y enfermedad de Sjögren, quien presenta desde 2012 un deterioro gradual del lenguaje con mayor progreso de los síntomas desde el año 2015.

El objetivo del caso es describir la progresión del perfil neurocognitivo de IA hasta el 2017, el cual se caracteriza por obtener un resultado esperado en orientación en tiempo y espacio, atención visual sostenida, praxia constructiva, gnosias visualesm, y un resultado inferior en lenguaje, lentificación en procesamiento de información, fallas en memoria verbal, visual y un deterioro en las funciones ejecutivas, perfil descrito por Butts et al. (2015) en 91 pacientes con las 3 variantes de APP.

La descripción de las características del lenguaje de IA, en la última evaluación realizada en el 2017, muestra una alta afectación en gramática, sintaxis, repetición de palabras y frases complejas, denominación de objetos, dificultad en encontrar palabras, construcción de frases, fallas en canto, ritmo y en el lenguaje espontáneo oral y escrito. Presenta un deterioro en la articulación, presentando parafasias fonémicas y circunloquios; la anterior descripción coincide con observaciones típicas de pacientes con APP no fluente, muy similares a una afasia de Broca (Kertesz \& Harciarek, 2014), así como la alteración en la repetición en oraciones largas se asocian a una afasia de conducción; igualmente, se puede observar en este caso lo reportado en pacientes de APPvnf que tartamudean al inicio de las palabras, hacen pausas en la mitad de la palabra, reducen la complejidad de la oración, cometen errores fonológicos, con baja producción, reducido contenido y una prosodia defectuosa (GornoTempini et al., 2011). Aún no se reflejan cambios en lectura ni en escritura al dictado ni a la copia, contrario a lo documentado por Dickerson (2011), quien describe un cambio progresivo entre la expresión oral y escrita.

En cuanto al diagnóstico diferencial entre las variantes, se descarta una $\mathrm{APPvS}$ debido a preserva la capacidad semántica, pero tiene alterada la fluidez, la articulación, la sintaxis y fonología mientras que pacientes con APPvS conservan. Adicionalmente, la correlación anatómica del caso no corresponde a la atrofia del polo anterior del lóbulo temporal izquierdo, característica de APPvS, por lo cual se descarta esta posibilidad diagnóstica (Gorno-Tempini et al., 2011).
En cuanto a la APPvl, se observa que el presente caso coincide en presentar parafasias fonémicas, alteración en la denominación, dificultad en la repetición de frases largas, con alteración en la memoria fonológica a corto plazo; pero, a diferencia de esta variante, IA no conserva la prosodia ni la gramática, lo cual si es conservado en los pacientes de la APPvl (Kertesz \& Harciarek, 2014). Respecto a esta variante, IA presenta una atrofia en la región parietal, similar a los aspectos morfológicos de la APPvl, en donde se afecta la región temporoparietal.

Los bajos resultados de la evaluación neuropsicológica de IA y de pacientes con afasia se pueden asociar a la cantidad de tiempo empleado en los test que generan agotamiento del paciente y del evaluador, especialmente en las baterías de evaluación del lenguaje (Kertesz \& Harciarek, 2014). Se ha descrito que en personas con APPvnf y APPvl los problemas de memoria en las pruebas de lista de palabras y el MMSE son bajos, debido a la dificultad en el procesamiento fonológico y la distorsión en el output verbal, por lo que frecuentemente son diagnosticados con demencia (Kertesz \& Harciarek, 2014).

Por esta razón, se sugiere que, en la evaluación neuropsicológica para pacientes con problemas severos de lenguaje, se usen pruebas estandarizadas no verbales como el Test Visuoverbal de funciones ejecutivas, test de Tres palabras y Tres formas para evaluar memoria y el test de orientación de líneas para las funciones visuoespaciales (Weintraub et al., 2013). Igualmente, se deben usar preguntas con opción de respuesta cerrada, preguntas cortas y simples (Sapolsky, Domoto-Reilly \& Dickerson, 2014).

En cuanto a la progresión del síndrome, IA cursa una fase de demencia afásica inicial, de evolución de 2 años al momento del estudio, tiempo en el que aparecen limitaciones más prominentes en otros dominios diferentes al lenguaje, como en memoria y funciones ejecutivas (Grossman, 2010). Respecto al lenguaje, se observa mayor afectación en denominación, reducción de palabras en las frases y esfuerzos para comunicarse. Esta fase puede durar varios años antes de la aparición de un estado de demencia avanzada. En estadios finales la APPvnf puede progresar hasta el mutismo, con conservación de la comprensión, lo que los diferencia a la afasia global, la demencia semántica y la EA. 
Los perfiles de progresión de los síntomas por subtipo tienen la ventaja de mostrar gravedad del deterioro del paciente, lo que puede ayudar en la planificación de la terapia del lenguaje incluyendo cuándo implementar el uso de dispositivos y estrategias de comunicación alternativas (Sapolsky, Domoto-Reilly \& Dickerson, 2014).

A nivel afectivo, la evaluada del caso no muestra cambios comportamentales, contrario a otros pacientes con APPvnf, quienes pueden desarrollar características de la variante comportamental de la DLFT, tales como desinhibición, hiperoralidad, cambios de alimentación o apatía (Gorno-Tempini et al., 2011).

La medida a través del PASS indica que la funcionalidad de IA en escenarios de poca facilitación, por parte de sus familiares, se ve afectada por la dificultad en la precisión de la articulación, la interrupción de la expresión oral debido a las pausas, así como la dificultad de encontrar la palabra adecuada en una conversación y fallas en la comprensión auditiva de oraciones largas; a diferencia de lo reportado por su esposo e hija, quienes la perciben con una alta funcionalidad, contemplándose aquí el sesgo existente en la valoración subjetiva con escalas versus las característica observadas y analizadas en ámbitos clínicos.

En conclusión, los déficits evidenciados en la paciente reúnen los criterios diagnósticos para un Trastorno neurocognitivo mayor frontotemporal, en estadio leve, sin alteración del comportamiento, características predominantes del tipo afasia progresiva primaria no fluente de acuerdo con su inicio y características de evolución.

La importancia de describir un perfil neuropsicológico de un caso de APP ayuda a los clínicos a identificar desde las fases iniciales hasta el diagnóstico de demencia afásica, los cambios en las características del lenguaje y en los otros dominios cognitivos, con el fin de dirigir los tratamientos e identificar tempranamente este tipo de síndromes.

\section{Limitaciones}

La evidente dificultad de las evaluaciones neuropsicológicas en pacientes afásicos radica en que están mediadas por el componente del lenguaje expresivo, por lo que limitó evaluar otros dominios. En cuanto a las pruebas utilizadas, algunas no se encuentran normalizadas para la población colombiana, ni traducidas al español, específicamente el PASS.

Cabe anotar que las escalas de funcionalidad en las actividades básicas e instrumentales en su mayoría implican acciones no lingüísticas (p. ej. Vestirse, ir al baño), por lo que se hizo necesario usar otros recursos para evaluar la funcionalidad específica y real en pacientes con afasia.

Finalmente, es importante tener en cuenta que la clasificación y características de trastorno neurocognitivo frontotemporal es reciente y aún se encuentra en estudio las características de las APP.

\section{Referencias}

Acosta, F., Canu, E., Sarro, L., Comi, G., \& Filipi, M. (2012). Neuroimaging findings in frontotemporal lobar degeneration spectrum of disorders. Cortex, 48, $389-413$.

Arango, J., \& Rivera, D. (2015). Neuropsicología en Colombia: Datos normativos, estado actual y retos a futuro. Manizales: Universidad Autónoma de Manizales.

Arnedo, M. Bembibre, J., \& Triviño, M. (2013). Neuropsicología a través de los casos clínicos. Editorial Médica Panamericana.

American Psychiatric Association. (2014). Guía de consulta de los criterios diagnósticos del DSM- 5 囚. American Psychiatric Publishing.

Barrero, S., García, A., Ojeda, M. (2005). Índice de Barthel (IB): Un instrumento esencial para la evaluación funcional y la rehabilitación. Plasticidad y Restauración Neurológica. 4(1-2), 81-85.

Beck, A., \& Steer, R. (2011). Manual. BAI. Inventario de Ansiedad de Beck (Adaptación española de Sanz, J.). Pearson Educación.

Brink, T. L. (1982). Development and validation of a geriatric depression screening scale: A preliminary report. Journal of Psychiatric Research.

Butts, A., Machulda, M., Duffy, J., Strand, E., Whitwell, J., \& Josephs, K. (2015). Neuropsychological Profiles Differ among the Three Variants of Primary Progressive Aphasia. Journal of the International Neuropsychological Society, 21 (6), 429-435. https:// doi.org/10.1017/S1355617715000399 
Dickerson, B. C. (2011). Quantitating Severity and Progression in Primary Progressive Aphasia. Journal of Molecular Neuroscience, 45(3), 618-628.

Goodglass, H., \& Kaplan, E., (1978). Test de Boston para el diagnóstico de la afasia. Editorial Medica Panamericana.

Gorno-Tempini, ML., Hillis, AE., Weintraub, S., Kertesz, A., Mendez, M., \& Cappa, SF. (2011). Classification of primary progressive aphasia and its variants. Neurology, 76, 1006-14.

Grober, E., Buschke, H., Crystal, H. A., Bang, S., \& Dresner, R. (1988). Screening for dementia by memory testing. Neurology, 38(6), 900-903.

Grossman, M. (2012). Primary progressive aphasia: clinicopathological correlations. Nature Reviews Neurology, 6, 88-97.

Lobo , A., Saz, P., Marcos, G., \& Grupo de Trabajo ZARADEMP.(2002) MMSE: Examen Cognoscitivo Mini-Mental. TEA Ediciones.

Harciarek, M., \& Kertesz, M. (2011). Primary Progressive Aphasias and Their Contribution to the Contemporary Knowledge About the Brain-Language Relationship. Rev. Neuropsychol Rev. 21:271-287. https://doi.org/10.1007/s11065-011-9175-9

Harris, J. M., \& Jones, M. (2014). Pathology in primary progressive aphasia syndromes. Current Neurology and Neuroscience Reports, 14, 8. https://doi. org/10.1007/s11910-014-0466-4

Kertesz, A., Nadkarni, N., Davidson W., \& Thomas A. W. (2000). The Frontal Behavioral Inventory in the differential diagnosis of frontotemporal dementia. J Int Neuropsychol Soc., 6(4), 460-8. https:// doi.org/10.1017/s1355617700644041. PMID: 10902415.

Kertesz, A., \& Harciarek, M. (2014). Primary progressive aphasia. Scandinavian Journal of Psychology, 55, 191-201.

Kertesz, A., \& Muñoz, D. G. (1998). Pick's disease and Pick complex. New York: Wiley-LISS.

Lawton, M. \& Brody, E. (1969). Assessment of older people: self-maintaining and Instrumental activities of daily living. Gerontologist, 9, 179-86.

Matías-Guiu, J. A., \& García-Ramos, R. (2013). Afasia progresiva primaria: del síndrome a la enfermedad. Neurología, 28 (6), 366-374.
Onyike, C. U., \& Diehl-Schmid, J. (2013). The Epidemiology of Frontotemporal Dementia. International Review of Psychiatry (Abingdon, England), 25(2), 130-137. https://doi.org/10.3109/09540261.20 13.776523.

Peña-Casanova, J. (1991). Programa integrado de exploración neuropsicológica - test barcelona: bases teóricas, objetivos y contenidos. Revista de Logopedia, Foniatría y Audiología, 11(1), 66-79. https:// doi.org/10.1016/S0214-4603(91)75505-8.

Peña-Casanova, J. (2007). Neurología de la conducta y Neuropsicología. Editorial Médica Panamericana.

Reitan, R. M., \& Wolfson, D. (1985). The Halstead-Reitan Neuropsychological Test Battery: Theory and Interpretattion. Tucson, AZ: Neuropsychology Press.

Rey, A. (1959). Test de Copie et Reproductione de Mémorie de Figures Géometriques Complexes. Adaptación española: Manual TEA 1980. 3a ed.

Rohan, Z., \& Matej, R. (2014). Current concepts in the classification and diagnosis of frontotemporal lobar degenerations: A practical approach. Archives of Pathology \& Laboratory Medicine, 138(1), 132-138.

Sapolsky, D., Domoto-Reilly, K., Negreira, A., Brickhouse, M., McGinnis, S., \& Dickerson, BC. (2014). Monitoring progression of primary progressive aphasia: current approaches and future directions. Neurodegenerative Disease Management, 1(1).

Seltman, R., \& Mattews, B. (2012). Frontotemporal Lobar Degeneration. Epidemiology, Pathology, Diagnosis and Management. CNS Drugs, 26(10), 841870.

Weintraub S, Rogalski E, Shaw E, Sawlani, S., Rademaker, A., Wieneke, C., \& Mesulam, M. (2013). Verbal and Nonverbal Memory in Primary Progressive Aphasia: The Three Words-Three Shapes Test. Behavioral Neurology, 26(1-2), 67-76. https:// doi.org/10.3233/BEN-2012-110239.

Wechsler, D. (2003). WAIS-III: Escala Wechsler de inteligencia para adultos-III. Ed. Manual Moderno. 